\title{
Study on Motions of a Floating Body under Composite External Loads
}

\author{
by Kunihiro Ikegami*, Member Masami Matsuura*, Member
}

\begin{abstract}
Summary
In the field of marine engineering, various types of floating bodies have been designed. Motions of them should be treated taking account of the effect of not only ocean waves but also external systems such as mooring lines, pipe lines, cables, or adjacent floating bodies.

Fundamental and systematic studies were carried out on motions of a floating body under composite external loads. Widely and commonly applicable prediction method was developed to predict them.

In order to verify the applicability of the prediction method, model experiments were carried out for the several typical cases of floating bodies under composite external loads. Fairly good agreements could be obtained between calculated and measured values, so that the availability of present prediction method was confirmed by experiments.

Where composite external loads can be approximated by linearized dynamic system, present method will be usefully applied to the design of various type of floating bodies under composite external loads.
\end{abstract}

\section{Introduction}

In the design of various marine structures and special purpose vessels such as dredgers, tugs, cable ships, research ships, etc., it is essentially important to predict the dynamical behaviours of them among waves, taking account of the influence of the loads such as mooring forces by mooring lines, tractions by cables or pipes, or connecting forces by connected floating bodies. These forces which are different from environmental forces due to waves, current, and wind, are called composite external loads in the present study. There exist many kinds of floating bodies and vessels with external loads as shown in Table 1. And, as a matter of fact, the behaviours of those floating bodies and vessels in waves are extremely complex.

In recent years, many studies have been carried out in regard to the motions of floating bodies under the composite external loads among waves $^{122) 32}$. However, most of them are for some particular kind of external loads; i.e., only for ordinary mooring lines, or only for towed cables, etc.

Present study has the intention, therefore, to investigate the problems fundamentally and

* Nagasaki Experimental Tank, M.H.I. systematically, and to develop the widely applicable method to estimate the characteristics of floating bodies and vessels among waves under multifarious external loads.

For a freely floating body, the prediction method of motions in waves has been widely established such as the strip method for ship motions. While, composite external loads can be regarded as the external dynamic system connected to a freely floating body. And for those external system also, many studies have been made elsewhere and prediction methods are available. Based on these established prediction methods of motions of a freely floating body and the external system, therefore, the motions of floating bodies under composite external loads can be estimated.

Treatment in the dynamics of a floating body can be classified into two cases as follows.

(I) Single-body problems: Motions of floating bodies are treated as the single-body problems considering the influence of the reaction forces and moments applied through the connecting points between a floating body and external loads.

(II) Multi-body problems: Motions of floating bodies are treated as the multi-body problems considering 
Table 1 Classification of floating bodies under composite external loads

\begin{tabular}{|c|c|c|}
\hline Type & Floating Dodies and vessels & Specific feature of composite external loads \\
\hline I & $\begin{array}{l}\text { Drilling platform (mooring line) } \\
\text { Floating storage tank (mooring line) } \\
\text { Dredger (suction pipe) } \\
\text { Pipe lay barge (pipe line) } \\
\text { Cable layer (cable) } \\
\text { Geological survey ship (towing cable) } \\
\text { Ocean mining ship (lift pipe) }\end{array}$ & $\begin{array}{l}\text { Single-body problems considering the } \\
\text { influence of the reaction forces and moments } \\
\text { applied through the connecting points } \\
\text { between a floating body and external loads. }\end{array}$ \\
\hline II & $\begin{array}{l}\text { Pusher barge system } \\
\text { Pin-jointed vessel } \\
\text { Floating oil fence } \\
\text { Multi-hull ship }\end{array}$ & $\begin{array}{l}\text { Multi-body problems considering the motions } \\
\text { of adjacent or connected bodies. }\end{array}$ \\
\hline$I+I I$ & $\begin{array}{l}\text { Floating breakwater } \\
\text { Multi-buoy system for collision protection } \\
\text { Buoy-ship mooring system }\end{array}$ & Compound case of both I and II. \\
\hline
\end{tabular}

the motions of adjacent or connected bodies.

The moored floating bodies are the typical examples for (I), and the pin-jointed vessel and pusher barge system are for (II). In order to treat the motions of moored and connected floating bodies such as floating breakwater, it is necessary to treat them as the combination of type (I) and (II).

The characteristics of external loads in general are non-linear such as the case for mooring line characterized by catenary theory. They can, however, be treated as a linear system on assumption that amplitudes of waves and motions of floating bodies are sufficiently small compared with their main dimensions. This linearlization of external loads appears to be reasonable for many a practical use.

In the present study, the prediction method was developed of linear motions of floating bodies under multifarious external loads among regular waves, based on the strip method widely used for the prediction of ship motions. And several model tests in waves were carried out for typical cases of moored or connected floating bodies so that the applicability of present prediction method were confirmed experimentally. Present paper describes an outline of the study.

\section{Prediction method of motions in waves}

Basic considerations are made in this chapter on a floating body system composed of multiple bodies connected and moored which is one typical configuration of a floating body under composite external loads. It is assumed that oscillatory motions of a floating body in waves are linear and harmonic, and connecting or mooring members are of negligible mass and also are treated as a linear dynamic system.

In the prediction of motions of multiple connected bodies, not only mutual actions between bodies through connecting members but also hydrodynamic interactions must be taken into consideration. It can be understood that the mutual interaction due to connecting members is a mechanical connection, and hydrodynamic interaction is a hydrodynamic connection. Therefore, both effects of them can be analyzed essentially by the same way in an analytical formulations of motions of the floating bodies.

\subsection{Equation of motions}

Analytical procedure for prediction of motions of a body freely floating among waves is the basis of the present problem, and for that procedure the strip method ${ }^{4}$ was adopted. The six-degree-of-freedom motions are defined as shown in Fig. 1. On assumption that the motions are linear and harmonic, the equation of motions

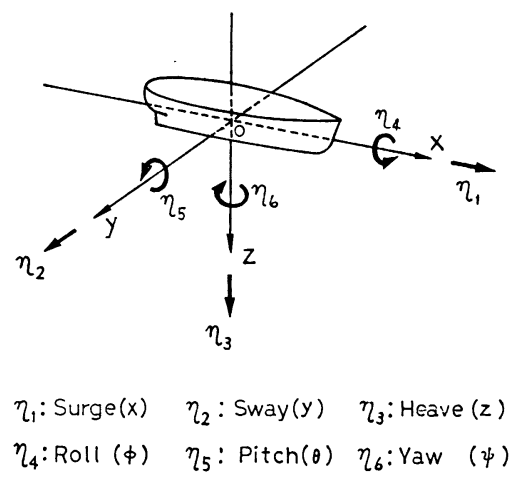

Fig. 1 Definition of six-degree-of-freedom motion 
for a single body freely floating in waves is given as follows in matrix notations:

$$
\left[-\omega_{e}^{2}(\boldsymbol{M}+\boldsymbol{A})+i \omega_{e} \boldsymbol{B}+\boldsymbol{C}\right] \boldsymbol{\eta}_{a}=\boldsymbol{F}_{a}
$$

where

$\boldsymbol{M}$ : generalized mass matrix for body $; 6 \times 6$ matrix

$\boldsymbol{A}$ : added-mass coefficients; $6 \times 6$ matrix

$\boldsymbol{B}$ : damping coefficients; $6 \times 6$ matrix

$\boldsymbol{C}$ : hydrostatic restoring coefficients; $6 \times 6$ matrix

$\eta$ : translatory and angular displacements of body ( $\left.=R_{e}\left[\eta_{a} e^{i \omega e t}\right]\right) ; 6 \times 1$ vector

$\boldsymbol{F}$ : wave exciting force and moment $\left(=R_{e}\left[\boldsymbol{F}_{a} e^{i \omega e t}\right]\right) ; 6 \times 1$ vector

$\omega_{e}$ : circular frequency of encounter

$a$ : subscript indicating complex amplitude

$j$ : subscript indicating mode of motion $(=1, \ldots, 6)$

Defining the linear operator $\boldsymbol{D}$ as follows:

$$
\boldsymbol{D}=-\omega_{e}^{2}(\boldsymbol{M}+\boldsymbol{A})+i \omega_{e} \boldsymbol{B}+\boldsymbol{C}
$$

the equation (1) may be simplified in the form:

$$
D \eta_{a}=F_{a}
$$

Extending the equation (3), the equation of motions for two bodies moored and connected each other is given as follows:

$$
\begin{aligned}
& {\left[\begin{array}{l:l}
\boldsymbol{D}_{1}+\boldsymbol{D}_{11}+\boldsymbol{H}_{1}+\boldsymbol{E}_{11} & \boldsymbol{D}_{12}+\boldsymbol{E}_{12} \\
\hdashline \boldsymbol{D}_{21}+\boldsymbol{E}_{21} & \boldsymbol{D}_{2}+\boldsymbol{D}_{22}+\boldsymbol{H}_{2}+\boldsymbol{E}_{22}
\end{array}\right]\left[\begin{array}{c}
\eta_{1 a} \\
\eta_{2 a}
\end{array}\right]} \\
& =\left[\begin{array}{c}
\boldsymbol{F}_{1 a} \\
\hdashline \boldsymbol{F}_{2 a}
\end{array}\right]
\end{aligned}
$$

where

$D_{l}$ : coefficients for a single body freely floating; $6 \times 6$ matrix

$\boldsymbol{D}_{l m}$ : hydrodynamic coefficients due to hydrodynamic interaction among multiple bodies; $6 \times 6$ matrix

$\boldsymbol{H}_{l}$ : added coefficients due to mooring members; $6 \times 6$ matrix

$\boldsymbol{E}_{l m}$ : added coefficients due to connecting members; $6 \times 6$ matrix

$l$ or $m$ : subscripts indicating quantities related to number of body ( $=1$ or 2 )

The equation (4) is in the case of two-body problem, but they can be easily expanded for multibody problems. On the assumption that the responses of multiple bodies are linear and harmonic, the added coefficients matrices $\boldsymbol{D}_{l m}$ or $\boldsymbol{E}_{l m}$ can be obtained from the forces acting on the $l$-th body in the case that the $m$-th body is forced to oscillate and the other bodies are restrained. Then, the wave exciting force must be calculated including the hydrodynamic interaction among multiple bodies.

2.2 Added coefficients matrix due to mooring and connecting members ${ }^{5}$

As shown in Fig. 2, a floating body is assumed

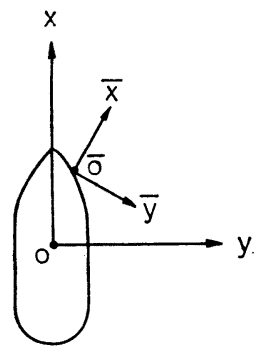

Fig. 2 Coordinate system for a moored body

to be moored at the point $\bar{o}$. The matrix $\boldsymbol{K}$ representing the characteristics of the mooring member is defined as follows in reference to the local coordinate system $\bar{o}-\bar{x} \bar{y} \bar{z}$.

$$
\boldsymbol{K}=\left[K_{j k}\right] ; j, k=1, \cdots, 6
$$

The element $K_{j k}$ of the matrix $K$ is the $j$-th mode force acting on the mooring member obtained from the $k$-th mode forced oscillation of the member at the point $\vec{o}$ jointing to a body. The matrix $\boldsymbol{K}$ is a $6 \times 6$ matrix in general, but it is reduced to a $3 \times 3$ matrix when a member does not transmit moments as in the case for an ordinary mooring lines. In the case that forces or moments acting on the mooring member are caused by not only deflection but velocity or acceleration of the jointed point, the matrix $\boldsymbol{K}$ may be of the same form as the matrix $\boldsymbol{D}$ as shown in the equation (2) considering damping and inertia forces as well as restoring forces. Defining the transformation matrix $\boldsymbol{T}$ (see Appendix) for transformation from the coordinate system $o-x y z$ to $\bar{o}-\bar{x} \bar{y} \bar{z}$ in Fig. 2, the added coefficients matrix $\boldsymbol{H}$ due to a mooring member are given as follows:

$$
\boldsymbol{H}=\boldsymbol{T}^{t} \boldsymbol{K} \boldsymbol{T}
$$

where the superscript $t$ is referred to the transpose of the matrix.

Next, it is supposed that the two bodies are connected together at the points $\bar{o}_{l}$ and $\bar{o}_{m}$ as shown in Fig. 3. It is assumed that the trans-

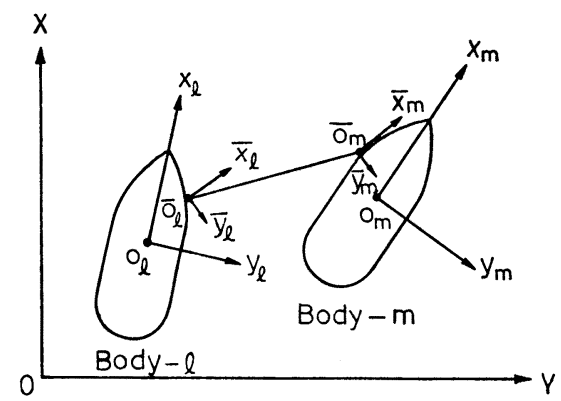

Fig. 3 Coordinate system for connected bodies 
formation matrices $\boldsymbol{T}_{l}$ and $\boldsymbol{T}_{m}$ can be defined for the transformation from the coordinate systems $o_{l}-x_{l} y_{l} z_{l}$ to $\bar{o}_{l}-\bar{x}_{l} \bar{y}_{l} \bar{z}_{l}$, and from $o_{m}-x_{m} y_{m} z_{m}$ to $\bar{o}_{m}-\bar{x}_{m} \bar{y}_{m} \bar{z}_{m}$, respectively, where in the coordinate systems $\bar{o}_{l}-\bar{x}_{l} \bar{y}_{l} \bar{z}_{l}$ and $\bar{o}_{m-}$ $\bar{x}_{m} \bar{y}_{m} \bar{z}_{m}$, the one is a parallel translation of the other. Similarly for the case of a moored body, the added coefficients matrices $\boldsymbol{E}$ are expressed as follows:

$$
\left.\begin{array}{l}
\boldsymbol{E}_{l l}=\boldsymbol{T}_{l}{ }^{t} \boldsymbol{K} \boldsymbol{T}_{l} \\
\boldsymbol{E}_{l m}=-\boldsymbol{T}_{l}{ }^{\imath} \boldsymbol{K} \boldsymbol{T}_{m} \quad(l \neq m)
\end{array}\right\}
$$

where the matrix $\boldsymbol{K}$ describes the characteristics of a connecting member.

\subsection{Hydrodynamic interaction among multiple bodies}

The approximate theoretical method for the calculation of hydrodynamic forces acting on multiple bodies was developed by Ohkusu'b7)8), and it is available for the present study. When the two-dimensional radiation problem for each body composing the multiple bodies is solved beforehand, the two-dimensional hydrodynamic forces are easily derived including the interaction among multiple bodies. Further, applying the strip method, the three-dimensional added hydrodynamic coefficients $\boldsymbol{D}_{l m}$ and wave exciting forces $\boldsymbol{F}_{l a}$ can be obtained which are required for deriving the solutions of motions including the effect of the hydrodynamic interaction. However, from the present status of the theory, the use of this method is restricted to the case of multiple bodies of an alongside configuration with zero forward speed in beam waves. As for the general case in oblique waves with forward speed, further theoretical and experimental investigations are necessary, especially for the evaluation of wave exciting forces in oblique waves.

\section{Model test}

In order to confirm the validity of present prediction method, model tests in waves were carried out for several kinds of moored or connected floating bodies in the Seakeeping and

\section{Case-1}

with spring in the vertical direction

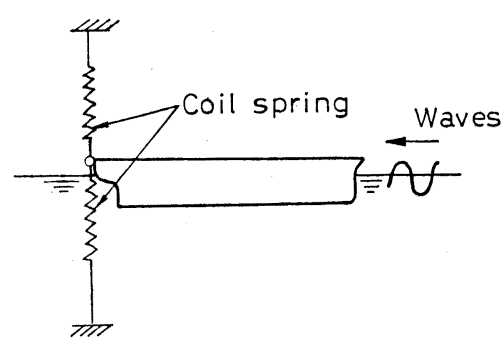

Case-2

with spring in the horizontal

direction

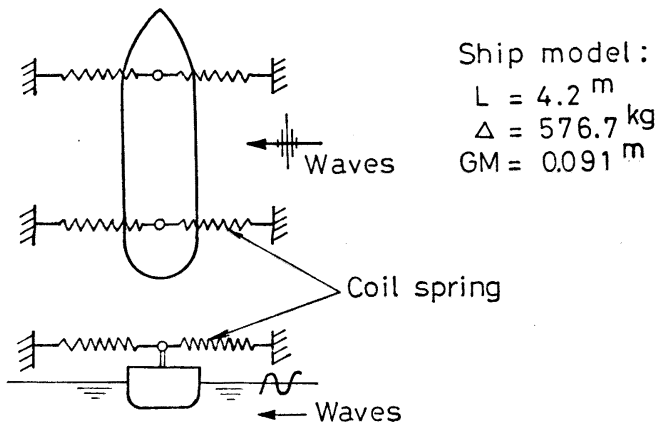

Fig. 4 Test arrangement for a ship moored by linear springs

\begin{tabular}{|c|c|c|c|}
\hline Condition & Measured & Computed & $h w / L$ \\
\hline without spring & 0 & & \\
\hline with spring & $\triangle$ & --- & \\
\hline
\end{tabular}
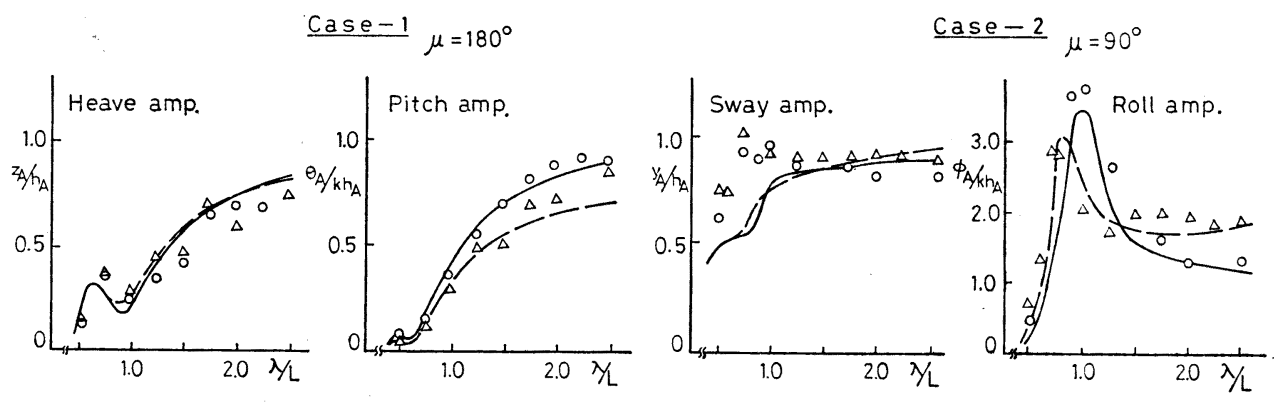

Fig. 5. Motions of a ship moored by linear springs 
Manoeuvring Basin of Nagasaki Experimental Tank, M.H.I.

\subsection{Floating body moored by linear spring}

As the case of the simplest external load, model test in wayes was carried out with linear spring in vertical and in horizontal direction, respectively. Principal particulars of tested model and test condition are shown in Fig. 4. Restoring moment due to spring was adjusted to 20 percent of hydrostatic pitching moment in vertical direction, and 20 percent of rolling moment in horizontal direction, respectively.

Some examples of the test results are shown in Fig. 5 in comparison with the calculated values. As shown in these figures, fairly good agreement is obtained between calculated and measured values. The influence of spring in vertical direction is remarkable in pitching motion and amplitude of motion becomes smaller. Under the influence of spring in horizontal direction, the natural rolling period becomes shorter, and the rolling amplitude at the synchronous point tends to be smaller. In the wave length range from $\lambda / L=1.5$ through 2.5, the amplitude tends to be larger, by the coupling effect of swaying motion.

\subsection{Two floating bodies connected with semi-} rigid link

For the case of connected floating bodies, model test in waves was carried out with two ship models connected by four semi-rigid links. The principal particulars of ship models and the arrangement of test are shown in Fig. 6. As shown in Fig. 7 connecting link model has only axial stiffness, of which spring constant is $0.1 \mathrm{~kg} / \mathrm{mm}$.

Some examples of test results for ship motions and axial displacement of links are shown in Figs. 8 and 9 in comparison with the calculated values. In beam sea condition, the calculations

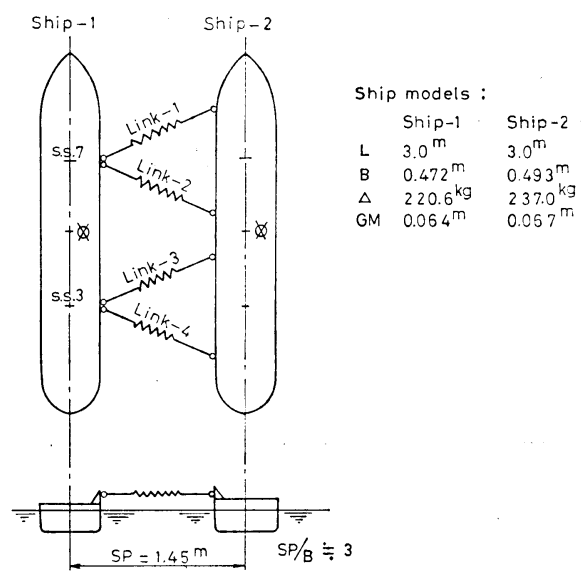

Fig. 6 Test arrangement for connected ships

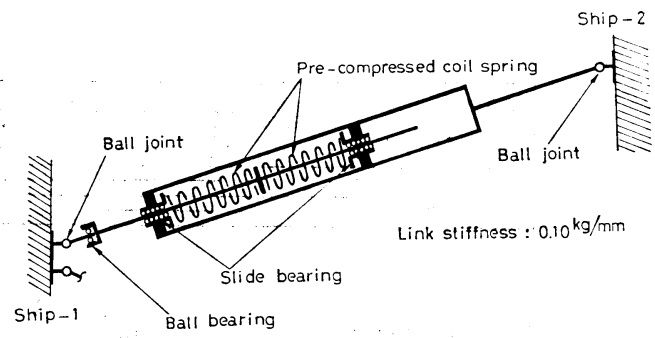

Fig. 7 Connecting link model with axial stiffness

were made with and without the hydrodynamic interactions between two ships.

By the influence of connecting link and hydrodynamic interaction, very complex coupled motions of two ships are caused, which differ from the motions of independent ships. The influence of connection is especially significant in the coupling of swaying and rolling motions. In shorter wave length range, the influence of hydrodynamic interaction is not so remarkable in experiments as in calculations. Effect of interaction by connecting link seems to be dominant compared with that of hydrodynamic interaction.

It is seen that fairly good agreement can be obtained between calculated and measured values, even when the influence of hydrodynamic interaction is neglected in the calculations.

\subsection{Floating bodies connected rigidly}

Setting the stiffness of connecting member to be very high, the present calculation method can be applied to floating bodies rigidly connected like catamaran. Model test in waves was carried out for two semi-immersed circular cylinders connected rigidly. The scheme of measuring arrangement and the principal particulars of tested model are shown in Fig. 10.

Some examples of test results are shown in Fig. 11 in comparison with the calculated values. In beam sea condition, the calculations were carried out with and without hydrodynamic interaction. For the rest of the wave incident angles, the calculations were made without hydrodynamic interaction.

In shorter wave length range of beam sea condition, the effect of hydrodynamic interaction on motions is remarkable. In this range, therefore, the hydrodynamic interaction should not be neglected in prediction of motions of catamaran. In other wave conditions, however, fairly good agreement can be obtained between calculated and measured values, even though the hydrodynamic interaction is neglected in calculation. 


\begin{tabular}{|c|c|c|c|}
\hline $\begin{array}{l}\text { Wave } \\
\text { direction }\end{array}$ & Measured & \multicolumn{2}{|c|}{ Computed } \\
\cline { 3 - 4 } & $\begin{array}{l}\text { without hydro. } \\
\text { interaction }\end{array}$ & $\begin{array}{l}\text { with hydro. } \\
\text { interaction }\end{array}$ \\
\hline$\mu=90^{\circ}$ & 0 & - & ----- \\
\hline $135^{\circ}$ & $\Delta$ & - & \\
\hline
\end{tabular}
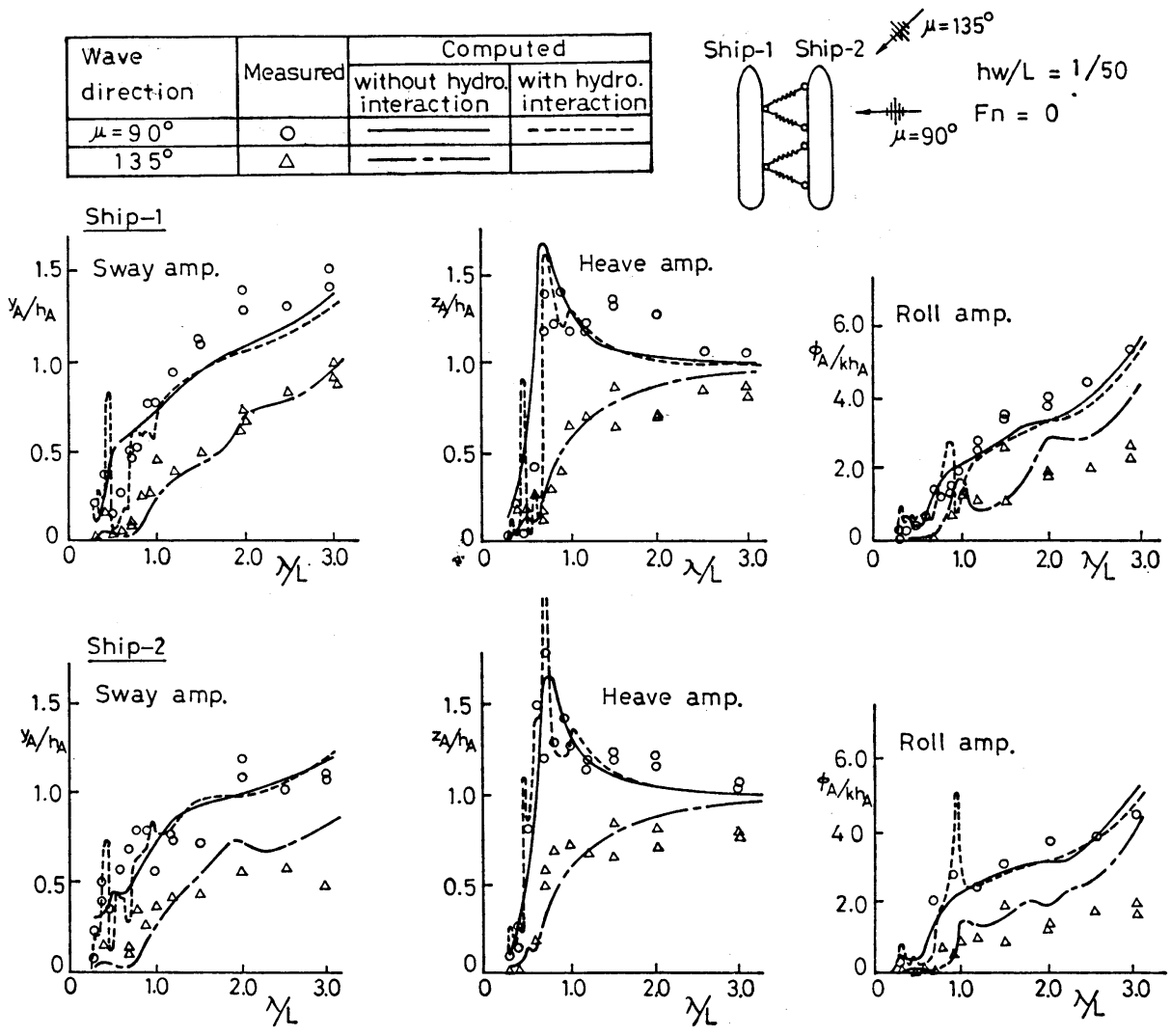

Fig. 8 Motions of connected ships
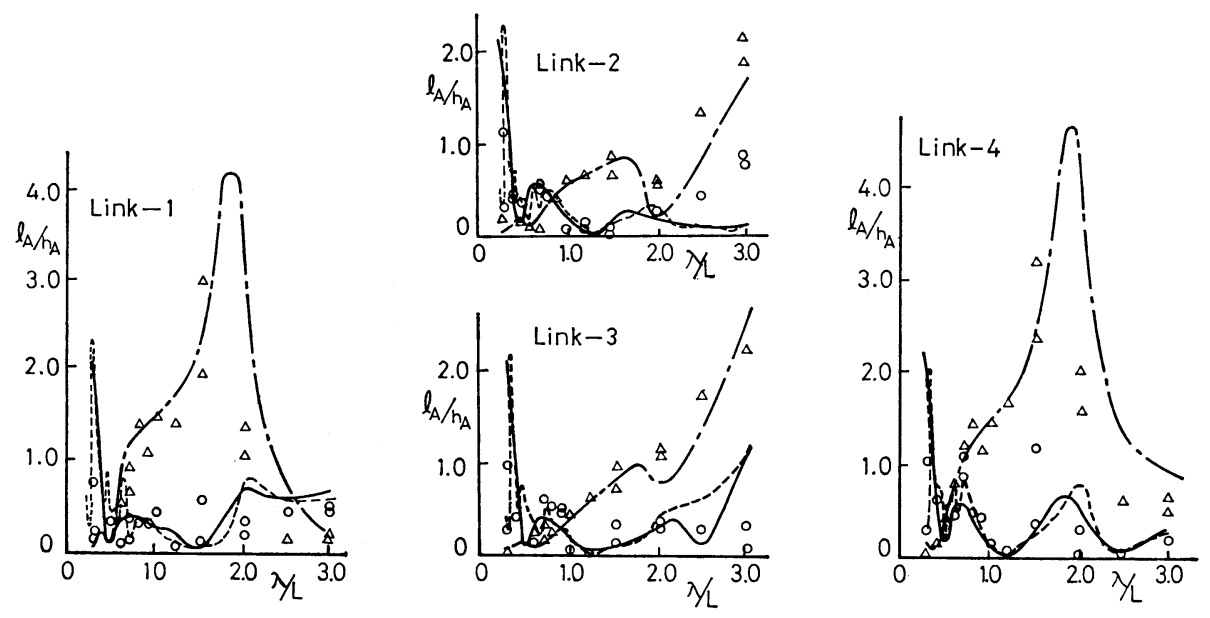

Fig. 9 Relative motions between connected ships 


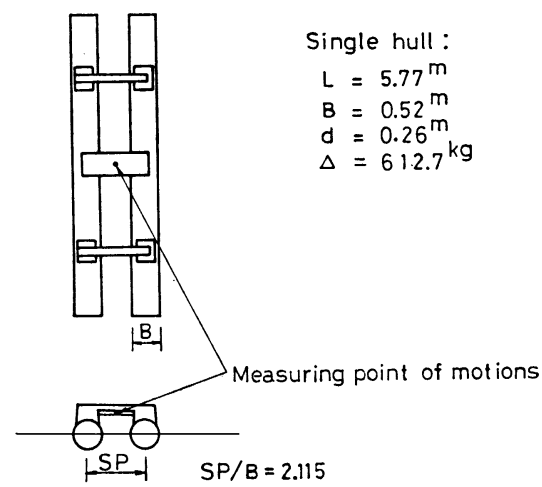

Fig. 10 Catamaran model composed of two semi-immersed horizontal circular cylinders

\begin{tabular}{|c|c|c|c|}
\hline Wave & \multirow{2}{*}{$\begin{array}{c}\text { Mirection } \\
\text { Measured }\end{array}$} & & \multicolumn{2}{|c|}{ Computed } \\
\cline { 3 - 4 } & $\begin{array}{l}\text { without hydro. } \\
\text { interaction }\end{array}$ & $\begin{array}{l}\text { with hydro. } \\
\text { interaction }\end{array}$ \\
\hline $90^{\circ}$ & 0 & - & ---- \\
\hline $120^{\circ}$ & 0 & - & \\
\hline $150^{\circ}$ & $\Delta$ & - & \\
\hline \multicolumn{3}{c}{$\mathrm{hw} / \mathrm{L}=1 / 100$} \\
$\mathrm{Fn}=0$
\end{tabular}
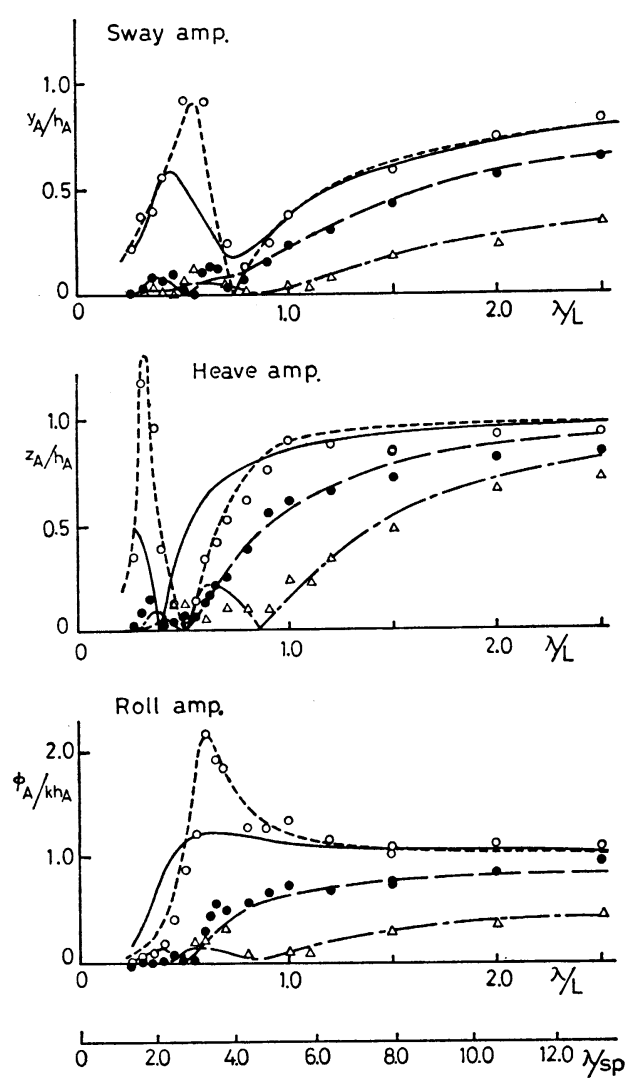

Fig. 11 Motions of catamaran cylinders

\section{Concluding remarks}

In the present paper, results of a series of systematic studies were described on motions of a floating body under composite external loads. And an analytical procedure for prediction on the basis of linear assumption was proposed. In order to verify the present prediction method model tests were carried out for the typical cases of the bodies with fundamental configurations.

The calculated values show satisfactory agreement with the experimental ones in general, so that the present method is confirmed fundamentally. So far as composite external loads can be replaced by linear dynamic systems, the method seems to have wide applicability to all type of 'connected', 'moored' or any other composite configurations consisting of dynamical systems of main body and additional ones including rigid connections as in catamaran hulls. Therefore, the theoretical calculation by use of the present method is useful for selecting configuration and connecting or mooring members of multiple bodies and for evaluation of those system in the initial stage of the design of the floating body.

Several problems, however, are left as the future works; i.e., linearization of connecting or mooring members with large non-linear property, rational prediction of hydrodynamic interaction in oblique waves and so on. For those problems, further theoretical and experimental studies will be necessary.

\section{Acknowledgement}

The authors wish to express their gratitude to Dr. H. Fujii, Mr. T. Takahashi and Mr. N. Toki of Nagasaki Technical Institute of Mitsubishi Heavy Industries, Ltd., for their stimulating and encouraging discussions in carrying out the present study. They also thank all members of Nagasaki Experimental Tank for their cooperation in carrying out the model tests.

\section{References}

1) Takagi, T., Arai, S., and Togano, Y.: A theoretical calculation of the motion of a moored floating vessel among the regular waves, J. Soc. Naval Arch. Japan, Vol. 135, (1974), p. 95.

2) Shoji, K.: Study on the motions of a moored body and the tension of mooring lines, J. Soc. Naval Arch. Japan, Vol. 138, (1975), p. 233.

3) Koterayama, W.: Motions of moored floating body and tension of mooring lines in waves, Trans. West-Japan Soc. Naval Arch. No. 53, (1977), p. 85. 
4) Salvesen, N., Tuck, E. O., and Faltinsen, O.: Ship motions and sea loads, Trans. SNAME, Vol. 78, (1970), p. 250.

5) Martin, H. C.: Introduction to matrix methods of structural analysis, McGrawHill, Inc., (1967).

6) Ohkusu, M.: On the motion of multihull ship in waves, Trans. West-Japan Soc. Naval Arch., No. 40, (1970), p. 19

7) Ohkusu, M. and Takaki, M.: On the motion of twin hull ship in waves, J. Soc. Naval Arch. Japan, Vol. 129, (1971), p. 29.

8) Ohkusu, M.: Ship motions in vicinity of a structure, Proc. Symp. on "Behaviour of Offshore Structure”, Norway, (1976).

\section{Appendix: Notes on the transformation of coordinates}

As shown in Fig. A-1, it is assumed that the vector of six-degree-of-freedom motions $\boldsymbol{\eta}$ of a rigid body is given in reference to the coordinate system $0-x y z$, and that the coordinate system $\bar{o}-\bar{x} \bar{y} \bar{z}$ is the parallel translation of $0-x y z$. And assumption is made that the motions are sufficiently small for linear approximation. The vector of motions $\eta$ which is transformed from $0-x y z$ to $\bar{o}-\bar{x} \bar{y} \bar{z}$, is given as follows by use of the transformation matrix $T_{p}$ :

$$
\bar{\eta}=T_{p} \eta
$$

where

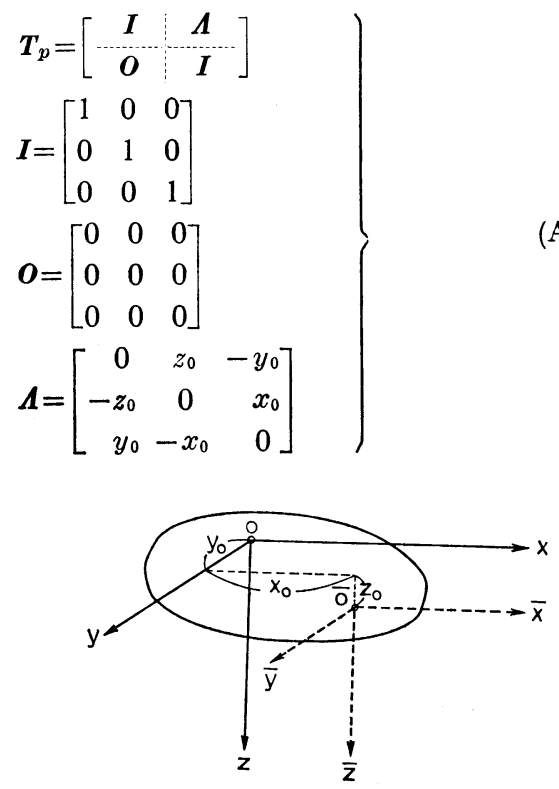

Fig. A-1 Parallel translation of coordinate

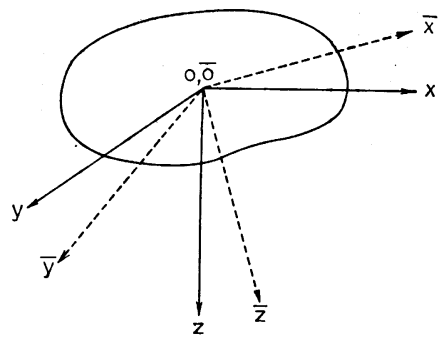

Fig. A-2 Rotation of coordinate

Using the same transformation matrix, the force $\overline{\boldsymbol{F}}$ acting upon the point $\bar{o}$ is transformed into the force $\boldsymbol{F}$ with respect to $o-x y z$ as follows.

$$
\boldsymbol{F}=\boldsymbol{T}_{p}{ }^{t} \overline{\boldsymbol{F}}
$$

Next, it is assumed that the coordinate system $\bar{o}-\bar{x} \bar{y} \bar{z}$ is a rotated system from $0-x y z$ as shown in Fig. A-2. Defining the transformation matrix $\boldsymbol{T}_{\boldsymbol{r}}$ for rotation of coordinate, the relation between the motions $\eta$ and $\bar{\eta}$, in reference to $o-x y z$ and $\bar{o}-\bar{x} \bar{y} \bar{z}$ respectively, is given as follows:

$$
\bar{\eta}=T_{r} \eta
$$

where

$$
\boldsymbol{T}_{r}=\left[\begin{array}{c:c}
\boldsymbol{\Gamma} & \boldsymbol{O} \\
\hdashline \boldsymbol{O} & \boldsymbol{\Gamma}
\end{array}\right]
$$

For instance, when the rotation is indicated by use of well-known Euler's angle $(\alpha, \beta, \gamma)$, the matrix $\Gamma$ in the equation (A-5) is given as follows.

$$
\begin{aligned}
\boldsymbol{\Gamma}= & {\left[\begin{array}{ccc}
\cos \gamma & \sin \gamma & 0 \\
-\sin \gamma & \cos \gamma & 0 \\
0 & 0 & 1
\end{array}\right]\left[\begin{array}{ccc}
\cos \alpha & 0 & -\sin \alpha \\
0 & 1 & 0 \\
\sin \alpha & 0 & \cos \alpha
\end{array}\right] } \\
& \cdot\left[\begin{array}{ccc}
\cos \beta & \sin \beta & 0 \\
-\sin \beta & \cos \beta & 0 \\
0 & 0 & 1
\end{array}\right]
\end{aligned}
$$

The forces $\boldsymbol{F}$ and $\overline{\boldsymbol{F}}$ have the similar relation to the equation $(\mathrm{A}-4)$ in reference to $o-x y z$ and $\bar{o}-$ $\bar{x} \bar{y} \bar{z}$ respectively. Namely,

$$
\boldsymbol{F}=\boldsymbol{T}_{r}{ }^{t} \overline{\boldsymbol{F}}
$$

In general, to transform the coordinate system $o-x y z$ into $\bar{o}-\bar{x} \bar{y} \bar{z}$ which both are the arbitrary coordinates fixed on a same rigid body, the transformation may be conducted in sequential order of parallel translation and rotation. Using the aforemensioned transformation matrices $\boldsymbol{T}_{\boldsymbol{p}}$ and $\boldsymbol{T}_{r}$, the transformation matrix of coordinate $\boldsymbol{T}$ as a whole is expressed as follows.

$$
T=T_{r} T_{p}
$$

